\title{
Renal Involvement in Retroperitoneal Fibrosis: Prevalence, Impact and Management Challenges
}

\section{Ichiro Mizushima \\ Mitsuhiro Kawano}

Department of Rheumatology, Kanazawa University Hospital, Kanazawa, Japan
Correspondence: Ichiro Mizushima Department of Rheumatology, Kanazawa University Hospital, I3-I, Takara-machi, Kanazawa, Ishikawa, 920-8640, Japan

Tel +8I-76-265-2253

Fax +8I-76-234-425I

Email ichiro7753@yahoo.co.jp

\begin{abstract}
Retroperitoneal fibrosis (RPF) is a rare disorder consisting of idiopathic and various secondary forms and characterized by chronic inflammatory infiltrates and marked fibrosis in the retroperitoneal space. In idiopathic RPF (IRPF), 35-60\% of cases have been reported to be IgG4-related RPF, the retroperitoneal lesions of IgG4-related disease (IgG4RD). IRPF can frequently lead to renal insufficiency mediated by urinary tract obstruction and hydronephrosis irrespective of being IgG4-related or not. Clinical pictures, laboratory and imaging findings, and location of the urinary tract obstruction are generally similar in IgG4-related and non-IgG4-related IRPF although multiple organ involvement and serum IgG4 elevation may be characteristic of the IgG4-related forms. Periaortic/periarterial lesions are the most frequent cause of renal insufficiency. Although the response to glucocorticoids is generally good, relapse does occur in a considerable proportion of patients, and may require an additional immunosuppressive agent and/or urological intervention in cases with multiple relapses or refractory obstructive uropathy. In general, the prognosis of patients with IRPF is good, but careful attention needs to be paid to chronic kidney disease as a major complication and rupture of the affected aorta/artery as a life-threatening one. Further studies are necessary to better understand the pathogenesis of the disease and to establish the optimal diagnostic and therapeutic strategies for it.
\end{abstract}

Keywords: retroperitoneal fibrosis, IgG4-related disease, hydronephrosis, renal involvement

\section{Introduction}

Retroperitoneal fibrosis (RPF) is a rare disorder characterized by chronic inflammatory infiltrates and marked fibrosis in the retroperitoneal space. ${ }^{1}$ Although the term "retroperitoneal fibrosis" has not been defined clearly, it encompasses a fibroinflammatory tissue surrounding the aorta and its major branches, also referred to as periaortitis/periarteritis, soft tissue masses in the renal pelvic wall and/or around the ureter, and placoid lesions in the pelvis and paravertebral area.

RPF consists of idiopathic and various secondary forms. ${ }^{1}$ Idiopathic RPF (IRPF) is considered to be an immune-mediated disorder. In IRPF, 35-60\% of cases have been reported to be immunoglobulin G4 (IgG4)-related RPF. ${ }^{2-5}$ Therefore, IRPF has recently been divided into IgG4-related RPF and non-IgG4-related IRPF although an obvious borderline between the two remains to be established. Indeed, RPF is one of the major organ manifestations of IgG4-related disease (IgG4-RD), which has become a widely recognized condition in recent years. Several large cohort studies have reported that the prevalence of RPF among overall patients with IgG4-RD is approximately $15-30 \% .^{6-10}$ On the other hand, secondary forms constitute about one-third of all RPF cases. ${ }^{11}$ Secondary etiologies of RPF 
include drugs (eg methysergide, ergot alkaloids, analgesics, beta-blockers, and dopamine agonists), infections (eg tuberculosis, histoplasmosis, and actinomycosis), malignancies (eg lymphoma, sarcoma, various metastatic carcinomas, and carcinoids), radiotherapy, trauma, major surgery of the abdomen, and proliferative disorders such as multicentric Castleman's disease and Erdheim-Chester disease. Therefore, an exclusion of various secondary etiologies is quite important in diagnosing IRPF or IgG4related RPF.

RPF frequently causes renal insufficiency mainly due to urinary tract obstruction. ${ }^{1}$ The location of the urinary tract obstructions and the lesions causing it vary widely. ${ }^{12}$ In addition, renal insufficiency can be caused by renal parenchymal lesions referred to as IgG4-related kidney disease (IgG4-RKD) $)^{13,14}$ especially in IgG4-related RPF. Such renal involvement in RPF can lead to not only acute kidney injury (AKI) but also chronic kidney disease (CKD) including renal atrophy or even end-stage renal disease (ESRD). ${ }^{15}$ Accordingly, the epidemiology, pathophysiology, clinical features, and management of RPF need to be clarified.

In this review, we provide an up-to date overview of IRPF and IgG4-related RPF, with particular emphasis on renal involvement in RPF.

\section{Epidemiology}

About $70 \%$ of all RPF cases have idiopathic forms, both IgG4-related and non-IgG4-related IRPF. The epidemiology of RPF has not yet been fully elucidated because few epidemiological studies are available. A case-control study in Finland estimated the prevalence and age-standardized incidence of IRPF to be 1.4 per 100,000 inhabitants and 0.10 per 100,000 person-years, respectively. ${ }^{16}$ A prospective study in the Netherlands showed a higher IRPF incidence of 1.3 per 100,000 person-years. ${ }^{17}$ IRPF occurs more frequently in persons aged 50-70 years and shows a male predominance with a male to female ratio of $2-4: 1{ }^{17-20}$ In observational studies of patients with IgG4-related $\mathrm{RPF}^{2-5,9}$ the mean age was 55.7 to 66.5 years, and the proportion of males was 55.5 to $100 \%$, both of which were consistent with those in overall IRPF cases.

\section{Risk Factors}

Risk factors for IRPF reported in the literature include asbestos exposure and smoking. ${ }^{16,21}$ A case-control study by Uibu et al showed that individuals exposed to asbestos were more than five times more likely to develop RPF. ${ }^{16}$
Another case-control study by Goldoni et al reported that combined exposure to asbestos and tobacco multiplicatively increased the odds ratio for developing IRPF. ${ }^{21}$ Interestingly, the association of smoking with IgG4-RD was shown in a recent case-control study, which additionally indicated the greater influence of current smoking on having IgG4-RD especially among patients with RPF compared with those without it. ${ }^{22}$

\section{Prevalence of Renal Involvement in RPF}

As mentioned above, urinary tract obstruction is a major and frequent presentation of renal involvement in RPF. In past reports limited to approximately 10 patients with IgG4-related $\mathrm{RPF}^{2-4,23}$ the prevalence of hydronephrosis and/or ureter obstruction was estimated to be $33-67 \%$. On the other hand, non-IgG4-related patients with IRPF had a comparable prevalence of hydronephrosis and/or ureter obstruction in the same studies, ${ }^{2-4}$ and IRPF patients with unknown relationship to IgG4 also had a comparable prevalence in other studies. ${ }^{24-30}$ Moreover, a larger series of 53 patients with IRPF showed that the prevalence of hydro-ureteronephrosis was $55 \% .{ }^{17}$ Therefore, the prevalence of urinary tract obstruction is high in IRPF irrespective of IgG4-related status.

Another cause of renal involvement in IRPF may be renal parenchymal lesions. In IgG4-RD, the kidney is one of the most frequently affected organs. The most common presentation is plasma cell-rich tubulointerstitial nephritis (TIN). The prevalence of renal lesions was reported to be approximately $10-20 \%$ of overall IgG4-RD cases. ${ }^{6-8,10}$ Only a few studies have investigated the prevalence of renal parenchymal lesions in IgG4-related RPF. A Japanese multicenter study investigating 99 patients with IgG4-related periaortitis/periarteritis and retroperitoneal fibrosis showed the prevalence of kidney lesion to be $16.2 \%{ }^{31}$ A Chinese retrospective study analyzing 58 patients with IgG4-related RPF among 407 with IgG4$\mathrm{RD}$ reported $25.9 \%$ of cases with kidney lesion in the 58 patients. ${ }^{9}$ On the other hand, non-IgG4-related IRPF rarely has extra-retroperitoneal lesions including renal ones. ${ }^{2,5,32}$ Accordingly, although rare, IgG4-related TIN should be brought to mind as a differential diagnosis in IRPF patients with renal insufficiency. In addition, it is also important to consider commonly occurring renal diseases in elderly patients, including diabetic kidney disease and hypertensive nephrosclerosis. 


\section{Clinical Pictures}

As clinical presentation of RPF, symptoms/signs such as abdominal (20-50\%), lower back (40-60\%), or flank (25$50 \%$ ) pain, lower extremity edema (2-20\%), low grade fever (5-15\%), appetite loss (2-15\%), weight loss (5-50\%), and decreased urinary excretion (4-5\%) are common. ${ }^{5,17-20}$ The pain in RPF is often difficult to localize, leading to substantial diagnostic delays, and its severity does not correlate with the presence of hydroureteronephrosis. Other urological manifestations including urinary frequency, testicular pain, varicocele, and hydrocele may also be observed. Rare vascular complications include deep venous thrombosis, lower limb claudication, and even pulmonary embolism.

In IgG4-related RPF, each of these clinical symptoms/ signs is observed at a comparable or lower frequency. ${ }^{31}$ In total, however, $58 \%$ of patients are asymptomatic, ${ }^{31}$ suggesting a more subclinical nature of IgG4-related RPF. In addition, any influence of the presence of hydronephrosis on the frequency of symptoms is also not evident. ${ }^{17,31}$ On the other hand, IgG4-related RPF more frequently shows other organ involvement, for which careful systemic screening is needed. ${ }^{5}$

\section{Laboratory Findings}

Usually, increased acute-phase reactants such as erythrocyte sedimentation rate (ESR) and C-reactive protein (CRP) are observed in patients with IRPF. Several studies reported that the levels of inflammatory markers were not significantly different between IgG4-related RPF and nonIgG4-related IRPF, ${ }^{4,5,32}$ while the frequency of serum CRP elevation was significantly higher in non-IgG4-related IRPF in one study. ${ }^{5}$ On the other hand, in patients with IgG4-RD, those with RPF showed significantly higher frequencies of elevated ESR and CRP levels compared with those without it. ${ }^{9}$ Kasashima et al suggested that not pelvic, paravertebral, and ureteral lesions but periaortic/ periarterial ones were associated with inflammatory features in IgG4-related RPF. ${ }^{33}$

Impaired renal function is another common finding in IRPF. Its frequency ranges from 33 to $71 \%$ of cases. ${ }^{17,19,20,25-29}$ IRPF patients with hydronephrosis had significantly higher serum creatinine levels than those without hydronephrosis. ${ }^{17}$ In patients with IgG4-RD, those with RPF showed significantly higher serum creatinine levels compared with those without RPF although the influence of hydronephrosis on renal function was not evaluated. $^{9}$ On the other hand, serum creatinine levels seem not to differ between IgG4-related and non-IgG4related IRPF. ${ }^{5,32}$

Hypocomplementemia is observed in some patients with IRPF. In IgG4-related RPF, the frequency of hypocomplementemia was reported to be approximately $25-30 \%, 5,31$ with a comparable frequency (22\%) noted in non-IgG4related IRPF. ${ }^{5}$ The impact of IgG4-RKD on hypocomplementemia was not analyzed in detail in these reports. In addition, hypocomplementemia was reported to be related to not only IgG4-RKD but also type 1 autoimmune pancreatitis (AIP) as a pancreatic lesion of IgG4-RD and IgG4related respiratory disease. ${ }^{10}$ However, the same report also demonstrated that severe hypocomplementemia was more strongly related to patients with IgG4-RKD than those with type 1 AIP or IgG4-related respiratory disease. ${ }^{10}$ Therefore, the finding of severe hypocomplementemia in IRPF, especially in the IgG4-related form, should prompt an evaluation of possible renal involvement. On the other hand, the mechanisms underlying hypocomplementemia in non-IgG4 -related IRPF remain to be further explored.

Anti-nuclear antibody (ANA) and other autoantibodies including rheumatoid factor (RF) and anti-thyroid antibodies are observed in some patients, whereas IRPF-specific autoantibodies have not been identified yet. In recent years, as IgG4-RD has become a more widely recognized condition, serum IgG4 levels are usually checked at the time of diagnosis. Indeed, one study defined IgG4-related periaortitis and idiopathic periaortitis based on the presence or absence of serum IgG4 elevation. ${ }^{32}$ However, we should consider that elevated serum IgG4 levels are observed in various other diseases as well, ${ }^{34}$ and that cases with IgG4-related RPF may have significantly lower serum IgG4 levels than other IgG4-RD cases without RPF. ${ }^{8}$ Therefore, serum IgG4 levels are of limited value in differentiating IgG4-related RPF from non-IgG4-related IRPF and other inflammatory or neoplastic disorders. On the other hand, because IRPF patients with high serum IgG4 levels more frequently have extra-retroperitoneal fibro-inflammatory lesions, ${ }^{35}$ an appropriate systemic screening for other potentially involved sites should be performed in such patients.

\section{Imaging Findings}

Some typical computed tomography (CT) and/or magnetic resonance imaging (MRI) findings of IRPF have been described $^{15}$ although they are not disease-specific, and a comprehensive approach considering clinical and radiological correlations is still needed. Compared with the findings 
in secondary RPF (eg malignancy), the typical CT/MRI findings of IRPF include soft tissue masses surrounding anterolaterally a infrarenal abdominal aorta (as opposed to malignant RPF which usually extends to a suprarenal aorta) and/or proximal common iliac arteries without displacing them, a tendency for the ureters to deviate medially (as opposed to lateral displacement by malignant RPF), and absence of infiltration into muscle or bone (Figure 1). In addition, contrast-enhanced CT and/or MRI show a characteristic delayed-phase homogeneous enhancement with paucity of enhancement in the early parenchymal phase.

Recently, the usefulness of positron emission tomography (PET)-CT in IRPF has been emphasized. ${ }^{36-38}$ PET-CT can identify extra-retroperitoneal involvement and is useful in monitoring disease activity after treatment. Its usefulness has similarly been reported in IgG4-RD. ${ }^{38,39}$

\section{Mechanisms Underlying Renal Involvement in RPF}

The location of the urinary tract obstructions and the lesions causing them vary widely in IRPF patients with hydronephrosis. Accordingly, it seems appropriate to divide the mechanisms of hydronephrosis into three categories. In addition, renal parenchymal lesions may also cause renal insufficiency in patients with IRPF. Here, based on a review of the past reports on IgG4-related RPF complicated by hydronephrosis ${ }^{12}$ and IgG4-RKD, ${ }^{40-42}$ we describe their respective characteristics as follows. In the review of IgG4-related RPF complicated by hydronephrosis, of 31 patients from the past case reports or case series, the properties of the lesions were described in 28 patients, and pre-treatment renal function was noted in $22 .{ }^{12}$ On the other hand, in the review of IgG4-RKD, the baseline patient data from 3 studies ${ }^{40-42}$ were reviewed although some patients were included in both studies. ${ }^{40,42}$

\section{Urinary Tract Obstruction or Stricture at the Renal Pelvis Due to Mass Lesion of the Renal Pelvis}

Hydronephrosis due to mass lesion of the renal pelvis or ureteropelvic junction was observed in 9 of 28 cases $(32.1 \%)$ in which the properties of the lesions were described. Six cases had left unilateral involvement, 2 right unilateral involvement, and one bilateral involvement. Of six cases whose pre-treatment renal function was noted, only one showed renal insufficiency with serum creatinine $>1.0 \mathrm{mg} / \mathrm{dL}$.

\section{Urinary Tract Obstruction or Stricture at the Upper to Lower Ureter Due to Encasement of the Ureter by Periaortic/ Periarterial Lesions}

Eleven of 28 cases (39.3\%) had ureter obstruction or stricture due to encasement by periaortic/periarterial lesions. The upper and middle ureters were the location of the obstruction or stricture in most cases. Five cases had bilateral
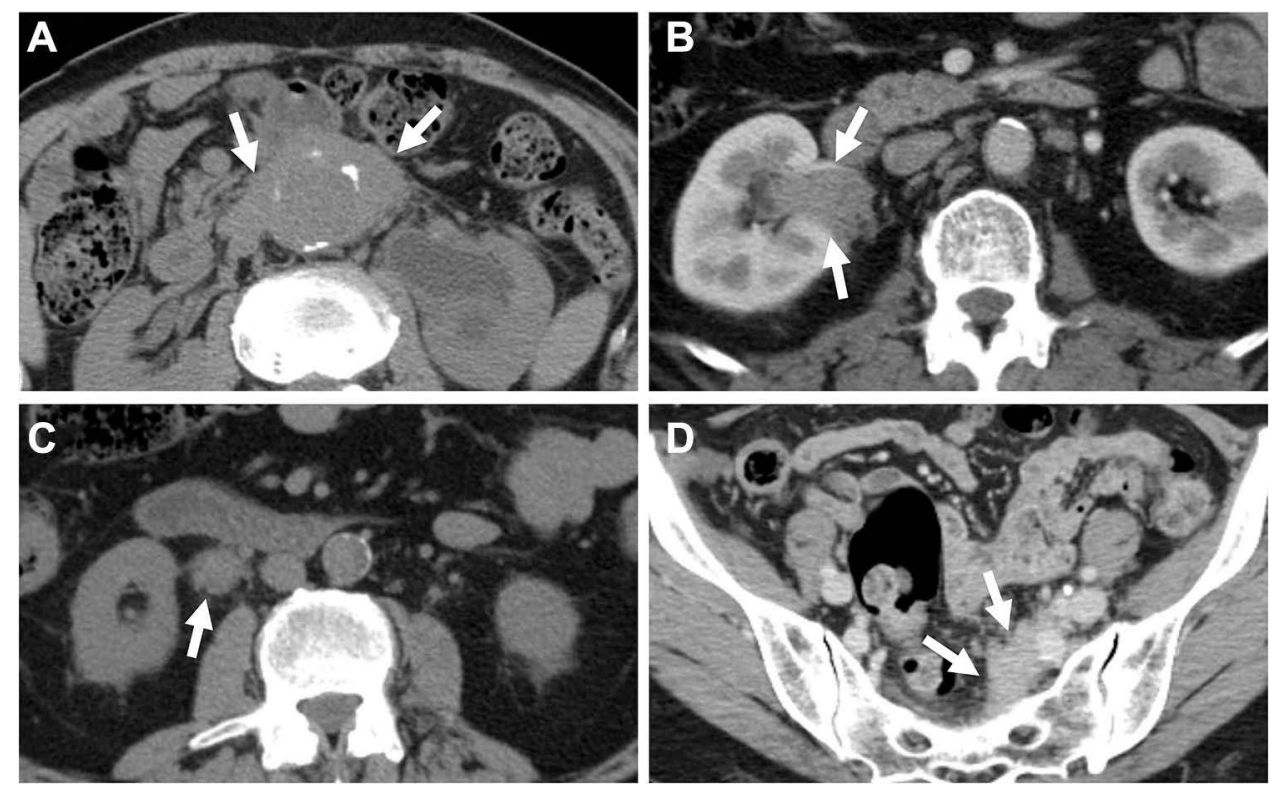

Figure I Typical computed tomography findings in idiopathic RPF. (A) Periaortic lesion (arrow) with aneurysmal change of the affected abdominal aorta and left hydronephrosis. (B) Right renal pelvic mass (arrow). (C) Right periureteral mass (arrow). (D) Placoid lesion (arrow) in the pelvis. 
involvement, and 3 each had left and right unilateral involvement, respectively. All ten cases whose pre-treatment renal function was mentioned had a serum creatinine exceeding $1.0 \mathrm{mg} / \mathrm{dL}$.

\section{Urinary Tract Obstruction or Stricture at the Upper to Lower Ureter Due to Periureteral Lesions}

Hydronephrosis due to concentric periureteral lesions or pseudotumor was detected in the remaining 8 cases $(28.6 \%)$. The location of the obstruction or stricture was distributed throughout the upper to lower ureter, with no obvious site predilection. Six cases had left unilateral involvement, and one each had right unilateral and bilateral involvement, respectively. Of six cases whose pretreatment renal function was noted, only one showed renal insufficiency with serum creatinine $>1.0 \mathrm{mg} / \mathrm{dL}$.

\section{Renal Parenchymal Lesions}

Due to the systemic nature of IgG4-RD, some patients with IgG4-related RPF may have IgG4-related TIN. In IgG4-related TIN, elevated serum creatinine levels $(>1.0 \mathrm{mg} / \mathrm{dL})$ are frequently observed at the time of diagnosis in $60-85 \%$ of cases. ${ }^{40-42}$ Characteristic radiologic findings include multiple low-density lesions, small cortical hypodense nodules, diffuse patchy involvement, and 'rim-like lesion of the kidney' on contrast-enhanced CT, and bilateral diffuse kidney enlargement despite severe renal dysfunction on plain $\mathrm{CT}$. These findings should be checked when unexplainable renal insufficiency without urinary tract obstruction is present in patients with IRPF. As mentioned above, because non-IgG4-related IRPF is rarely accompanied by extra-retroperitoneal fibroinflammatory lesions, these renal parenchymal lesions are not expected to be observed in non-IgG4-related IRPF.

Thus, from the review of published reports, the most frequent lesions causing renal involvement in RPF appear to be periaortic/periarterial lesions, which frequently cause bilateral hydronephrosis and renal insufficiency. Although these findings were found based on a review of IgG4-related forms, they may also apply to patients with non-IgG4related IRPF because of a lack of obvious differences in the location of the retroperitoneal lesions and prevalence of hydronephrosis between IgG4-related RPF and non-IgG4related IRPF. $^{2-5,32}$ Studies of larger numbers of patients are required to validate these findings.

\section{Management}

The main purpose of treatment for IRPF is to preserve renal function. Although obtaining and maintaining remission of the lesions would be ideal, the lesions without urinary tract obstruction or renal insufficiency can be treated with only medical therapy or even allowed to be carefully observed with neither medical therapy nor ureteral drainage especially when the patients show aneurysmal changes in the affected aorta/artery. In treating patients with AKI and/or at least moderate obstructive uropathy on imaging, ureteral drainage is necessary using ureteral stents or nephrostomies, followed by medical therapy. ${ }^{43,44}$ If patients are refractory to such combination therapy, a surgical approach, such as ureterolysis with omental fat wrapping of the ureters, is efficacious. Relapse does occur in a considerable proportion of patients $(15-50 \%),{ }^{25-29}$ leading to the necessity of long-term clinical monitoring and maintenance therapy in most patients. Accordingly, a conservative medical therapy with and without temporary ureteral stenting or percutaneous nephrostomy depending on the presence of progressive renal insufficiency or urinary tract obstruction is recommended, with surgery reserved for refractory cases (Figure 2).

The first-line medical therapy is glucocorticoids, with initial doses of $0.6-1 \mathrm{mg} / \mathrm{kg}$ per day of prednisolone gradually tapered to a maintenance dose of $2.5-5 \mathrm{mg} /$ day. ${ }^{45,46}$ In most cases, reduction of the retroperitoneal lesions and improvement of renal function are achieved in this way, 2,23,46,47 making possible stent removal in many of the patients who have been subjected to ureteral stenting. However, as mentioned above, relapses after tapering or cessation of glucocorticoids are often experienced in IRPF, as well as IgG4-RD which is well known to frequently relapse. Concerning the regimen of glucocorticoid therapy, several differences are noted between IgG4- and non-IgG4related forms of RPF. In the former, the initial prednisolone dose of $0.6 \mathrm{mg} / \mathrm{kg} / \mathrm{day}$, continuation of the initial dose for 2-4 weeks, and tapering by $5 \mathrm{mg}$ every $1-2$ weeks to a maintenance dose (2.5-5 $\mathrm{mg}$ /day) over a period of 2-3 months are recommended according to the standard glucocorticoid treatment for type 1 AIP. ${ }^{48}$ Moreover, in Japan, long-term maintenance glucocorticoid therapy for 3 years to decrease the number of relapses is recommended based on the results of several studies ${ }^{49,50}$ while a shorter duration of maintenance therapy is common in Western countries. On the other hand, in the latter, the initial prednisolone dose of 


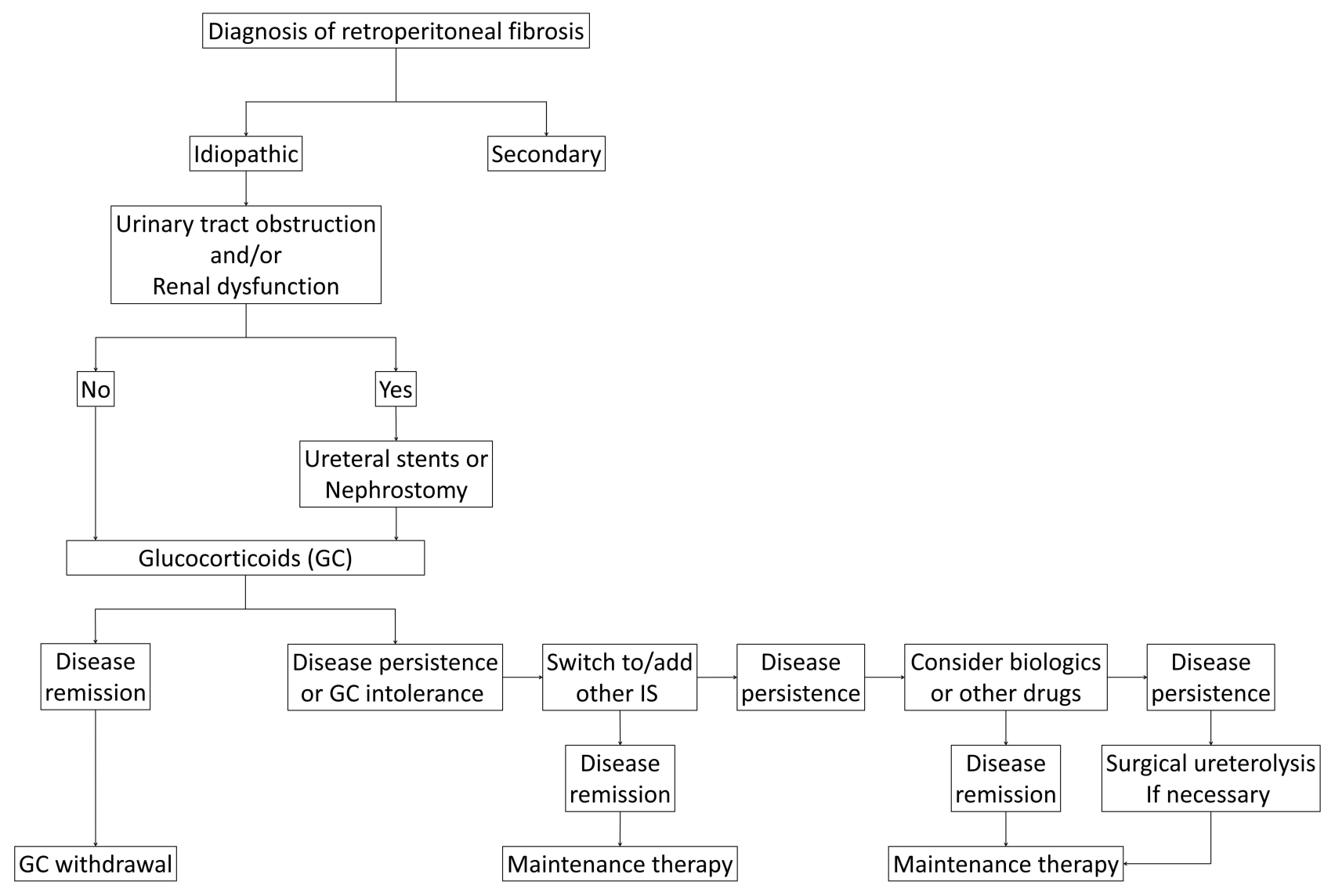

Figure 2 Proposed algorithm for the management of idiopathic RPF. Abbreviations: GC, glucocorticoids; IS, immunosuppressants.

$1 \mathrm{mg} / \mathrm{kg} /$ day for $1 \mathrm{month}$ and tapering to $10 \mathrm{mg} /$ day in the following 3 months, followed by a slow tapering for another 6 months until drug cessation, are recommended. ${ }^{51}$ The impact of these differences on the relapse rate and/or prognosis in each form remains to be clarified.

The efficacy of tamoxifen, monotherapy or in combination with glucocorticoids, in IRPF has been shown in several studies. ${ }^{52-54}$ In randomized controlled trial, however, maintenance treatment with tamoxifen after prednisone induction was significantly less effective for decreasing relapses compared with that with prednisone despite fewer glucocorticoid-associated side effects. ${ }^{55}$ Therefore, tamoxifen has been proposed as an alternative to glucocorticoids for IRPF patients with contraindications to glucocorticoids or intolerance for glucocorticoid toxicity. The use of tamoxifen for treatment of IgG4-related RPF or IgG4-RD has rarely been reported.

Other immunosuppressants reported as a valuable option for treatment of IRPF include methotrexate, ${ }^{56}$ cyclophosphamide, ${ }^{57}$ azathioprine, ${ }^{58}$ and mycophenolate mofetil ${ }^{59}$ although clinical evidence of these agents from controlled trial is lacking. In addition, refractory cases treated with biological agents such as rituximab ${ }^{60}$ and tocilizumab ${ }^{61}$ have been reported in case reports or case series, suggesting their efficacy. Interestingly, many of these immunosuppressive agents ${ }^{62-67}$ also show therapeutic efficacy for IgG4-RD. Especially, the efficacy of rituximab is notable in both IgG4-related RPF and non-IgG4-related IRPF. In recent retrospective studies, rituximab administration (1000 mg 2 weeks apart or $375 \mathrm{mg} / \mathrm{m}^{2} /$ week $\times 4$ weeks) with or without glucocorticoids achieved amelioration of symptoms, improvement of serum inflammatory markers, radiologic reduction of the retroperitoneal lesions, and dose reduction of glucocorticoids. ${ }^{68,69}$ Of note, rituximab monotherapy was as efficacious as combination therapy of rituximab and glucocorticoids against both IgG4-related RPF and non-IgG4-related IRPF, suggesting the possibility of effective treatment without glucocorticoid and its associated toxicity. ${ }^{68,69}$ Further evaluation of these agents is necessary to establish the most effective treatment strategy.

Ureteral drainage and/or surgical intervention are another important component in the management of RPF. 
As ureteral drainage, percutaneous nephrostomy and indwelling ureteral stents are both efficacious with comparable safety and can be removed under appropriate medical treatment ${ }^{44,70}$ although the latter has a higher incidence of obstruction during the indwelling period. ${ }^{44}$ Surgical intervention includes ureterolysis by open exploration or laparoscopy with omental fat wrapping of the ureters and intraperitoneal transposition, ${ }^{53,71,72}$ which is regarded as the best surgical approach to relieve ureteral obstruction. However, since surgical treatment does not prevent progression and recurrence of disease, and has no or little effect on the systemic manifestations, preceding medical therapy is recommended.

\section{Prognosis}

In general, the prognosis of patients with IRPF is good. Long-term observation studies reported mortality rates of $0-9 \%{ }^{25,26,53}$ and ESRD rates of $0-8 \%{ }^{25-27,29}$ although the follow-up durations were different among these studies (Table 1). On the other hand, CKD is a major complication of IRPF, and presumably results from urinary tract obstruction due to the retroperitoneal lesions. CKD defined as an estimated glomerular filtration rate (eGFR) of less than $60 \mathrm{~mL} / \mathrm{min} / 1.73 \mathrm{~m}^{2}$ was observed in $20-47 \%$ of IRPF patients. $^{25,26,29}$ The risk factors for these poor outcomes in IRPF have not been established partly because of inconsistencies in the treatment regimens and disease monitoring protocols between individual studies.

Relapse is another important outcome investigated in several past studies. As mentioned above, observation studies demonstrated that relapses during the observation periods were observed in approximately $15-50 \%$ of patients. $^{25-29}$ Although the suggested risk factors of relapse were inconsistent among the studies, they included higher baseline ESR, ${ }^{25}$ a shorter course of glucocorticoid treatment, ${ }^{25}$ ANA positivity, ${ }^{27}$ smoking, ${ }^{27} \mathrm{AKI},{ }^{27}$ and lumbar pain. ${ }^{27}$ Reliable predictors of relapses are desired because relapse is suggested to result in a greater eGFR decline during the clinical course. ${ }^{27}$

Long-term outcomes or relapse predictors specific for IgG4-related RPF have not been clarified yet. In comparison with non-IgG4-related IRPF, some studies showed a higher recurrence rate in IgG4-related $\mathrm{RPF},{ }^{4}$ while others demonstrated no significant differences in the response to therapy or relapses between the two group. ${ }^{35}$ On the other hand, several studies investigated relapse predictors of IgG4-RD not limited to IgG4-related RPF. In type 1 autoimmune pancreatitis (AIP), the pancreatic lesion of IgG4-
$\mathrm{RD}$, the risk factors for relapse were reported to include absence of low-dose maintenance glucocorticoid treatment, presence of IgG4-related sclerosing cholangitis, and high immune complex level in long-term observational studies or randomized controlled trials. ${ }^{49,50,73,74}$ Different factors including younger age, male gender, elevation of serum IgG4, IgG, and IgE levels, eosinophil counts, RF positivity, and initial dose of glucocorticoids were identified in long-term observational studies focusing on a large number of subjects from the whole IgG4-RD population mainly consisting of patients with dacryoadenitis and sialadenitis. ${ }^{75-77}$ In addition, our own recent study demonstrated that the risk factors of recurrent and de novo organ involvement were different in IgG4-RD. Risk factors of recurrent organ involvement were younger age and ANA positivity, whereas those of de novo organ involvement were elevation of peripheral blood eosinophil counts and discontinuation of glucocorticoids/observation without glucocorticoids. $^{78}$

Attention also needs to be paid to one particular treatment risk when patients with hydronephrosis due to periaortic/ periarterial lesions are treated. Early initiation of glucocorticoid therapy is desired for preservation of renal function, but careful follow-up after it is essential when the affected aorta/ artery has already shown luminal dilatation or aneurysmal formation. A risk of exacerbation of the existing luminal dilatation or rupture of the affected aorta/artery is presumed as an unintentional consequence of such treatment in IgG4related periaortitis/periarteritis ${ }^{47,79-81}$ (Figure 3).

\section{Conclusions}

IPRF both IgG4-related and non-IgG4-related is a rare disorder that frequently causes several types of renal involvement, mainly obstructive uropathy due to periaortic/ periarterial lesions. Not only AKI due to temporary urinary tract obstruction but also CKD of various severities in persistent cases can occur. However, appropriate management with immunosuppressive agents and urological interventions can improve the renal prognosis and prevent ESRD. Although the prognostic difference remains unclear between IgG4-related and non-IgG4-related forms, more attention should be paid to multiple organ involvement including IgG4-related TIN as another cause of renal insufficiency in IgG4-related RPF. In addition, exacerbation of the luminal dilatation or rupture of the affected aorta/artery may occur as an unintended consequence of treatment in IgG4-related RPF, and so requires careful follow-up observation. Further studies will be necessary to better clarify the 


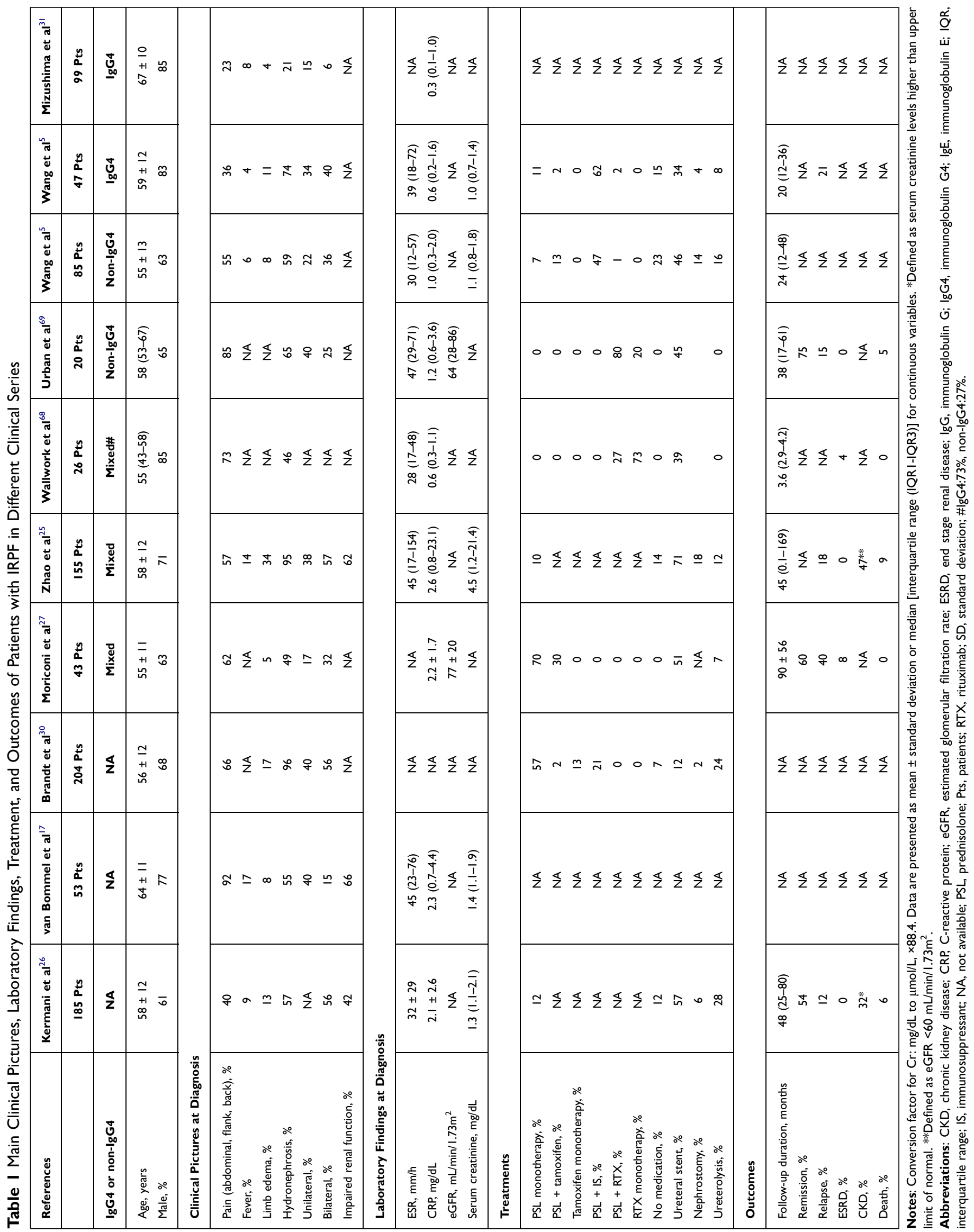



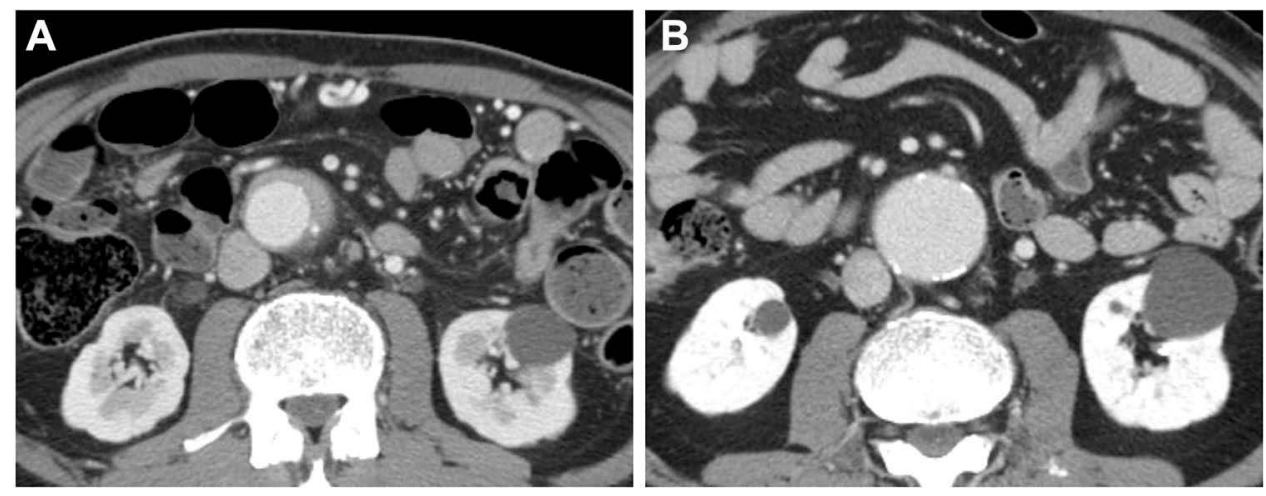

Figure 3 Aneurysm formation at the periaortic lesion of the abdominal aorta after glucocorticoid therapy. Before (A) and after (B) glucocorticoid therapy, the periaortic lesion is markedly ameliorated, while an aneurysm has developed at the same site.

pathogenesis of this disease and to establish optimal diagnostic and therapeutic strategies.

\section{Acknowledgments}

We thank John Gelblum for his critical reading of the manuscript.

\section{Funding}

There is no funding to report.

\section{Disclosure}

The authors report no conflicts of interest in this work.

\section{References}

1. Vaglio A, Salvarani C, Buzio C. Retroperitoneal fibrosis. Lancet. 2006;367:241-251. doi:10.1016/S0140-6736(06)68035-5

2. Zen $\mathrm{Y}$, Onodera $\mathrm{M}$, Inoue $\mathrm{D}$, et al. Retroperitoneal fibrosis: a clinicopathologic study with respect to immunoglobulin G4. Am J Surg Pathol. 2009;33:1833. doi:10.1097/PAS.0b013e3181b72882

3. Khosroshahi A, Carruthers MN, Stone JH, et al. Rethinking Ormond's disease: "idiopathic" retroperitoneal fibrosis in the era of IgG4-related disease. Medicine (Baltimore). 2013;92:82. doi:10.1097/ MD.0b013e318289610f

4. Koo BS, Koh YW, Hong S, et al. Clinicopathologic characteristics of IgG4-related retroperitoneal fibrosis among patients initially diagnosed as having idiopathic retroperitoneal fibrosis. Mod Rheumatol. 2015;25:194. doi:10.3109/14397595.2014.931908

5. Wang K, Wang Z, Zeng Q, et al. Clinical characteristics of IgG4-related retroperitoneal fibrosis versus idiopathic retroperitoneal fibrosis. PLoS One. 2021;16:e245601. doi:10.1371/journal. pone. 0245601

6. Zen Y, Nakanuma Y. IgG4-related disease: across-sectional study of 114 cases. Am J Surg Pathol. 2010;34:1812-1819. doi:10.1097/ PAS.0b013e3181f7266b

7. Inoue D, Yoshida K, Yoneda N, et al. IgG4-related disease: datasetof 235 consecutive patients. Medicine (Baltimore). 2015;94:e680. doi:10.1097/MD.000000000000680

8. Wallace ZS, Deshpande V, Mattoo H, et al. IgG4-related disease: clinicaland laboratory features in 125 patients. Arthritis Rheumatol. 2015;67:2466-2475. doi:10.1002/art.39205
9. Liu Y, Zhu L, Wang Z, et al. Clinical features of IgG4-related retroperitoneal fibrosis among 407 patients with IgG4-related disease: a retrospective study. Rheumatology (Oxford). 2021;60:767-772. doi:10.1093/rheumatology/keaa411

10. Yamada K, Yamamoto M, Saeki T, et al. New clues to the nature of immunoglobulin G4-related disease: a retrospective Japanese multicenter study of baseline clinical features of 334 cases. Arthritis Res Ther. 2017;19:262. doi:10.1186/s13075-017-1467-x

11. Cronin CG, Lohan DG, Blake MA, Roche C, McCarthy P, Murphy JM. Retroperitoneal fibrosis: a review of clinical features and imaging findings. AJR Am J Roentgenol. 2008;191:423-431. doi:10.2214/AJR.07.3629

12. Mizushima I, Inoue D, Kawano M. Retroperitoneal fibrosis/periaortitis and hydronephrosis. In: Saito T, Stone JH, Nakashima H, Saeki T, Kawano M, editors. Igg4-Related Kidney Disease. Tokyo: Springer; 2016:159-171.

13. Kawano M, Saeki T, Nakashima H, et al. Proposal for diagnostic criteria for IgG4-related kidney disease. Clin Exp Nephrol. 2011;15:615-626. doi:10.1007/s10157-011-0521-2

14. Capecchi R, Giannese D, Moriconi D, et al. Renal involvement in IgG4-related disease: from sunlight to twilight. Front Med. 2021;8:635706. doi:10.3389/fmed.2021.635706

15. Raglianti V, Rossi GM, Vaglio A. Idiopathic retroperitoneal fibrosis: an update for nephrologists. Nephrol Dial Transplant. 2020.gfaa083. doi:10.1093/ndt/gfaa083

16. Uibu T, Oksa P, Auvinen A, et al. Asbestos exposure as a risk factor for retroperitoneal fibrosis. Lancet. 2004;363:1422-1426. doi:10.1016/S0140-6736(04)16100-X

17. van Bommel EF, Jansen I, Hendriksz TR, et al. Idiopathic retroperitoneal fibrosis: prospective evaluation of incidence and clinicoradiologic presentation. Medicine (Baltimore). 2009;88:193-201. doi:10.1097/MD.0b013e3181afc420

18. Scheel PJ Jr, Feeley N. Retroperitoneal fibrosis: the clinical, laboratory, and radiographic presentation. Medicine (Baltimore). 2009;88:202-207. doi:10.1097/MD.0b013e3181afc439

19. Gómez García I, Sánchez Castaño A, Romero Molina M, et al. Retroperitoneal fibrosis: single-centre experience from 1992 to 2010, current status of knowledge and review of the international literature. Scand J Urol. 2013;47:370-377. doi:10.3109/ 00365599.2012.747564

20. Li KP, Zhu J, Zhang JL, et al. Idiopathic retroperitoneal fibrosis (RPF): clinical features of 61 cases and literature review. Clin Rheumatol. 2011;30:601-605. doi:10.1007/s10067-010-1580-6

21. Goldoni M, Bonini S, Urban ML, et al. Asbestos and smoking as risk factors for idiopathic retroperitoneal fibrosis: a case-control study. Ann Intern Med. 2014;161:181-188. doi:10.7326/M13-2648 
22. Wallwork R, Perugino CA, Fu X, et al. The association of smoking with IgG4-related disease: a case-control study. Rheumatology (Oxford). 2021.keab172. doi:10.1093/rheumatology/keab172

23. Chiba K, Kamisawa T, Tabata T, et al. Clinical features of 10 patients with IgG4-related retroperitoneal fibrosis. Intern Med. 2013;52:1545-1551. doi:10.2169/internalmedicine.52.0306

24. Raffiotta F, da Silva Escoli R, Quaglini S, et al. Idiopathic retroperitoneal fibrosis: long-term risk and predictors of relapse. $\mathrm{Am}$ J Kidney Dis. 2019;74:742-750. doi:10.1053/j.ajkd.2019.04.020

25. Zhao J, Li J, Zhang Z. Long-term outcomes and predictors of a large cohort of idiopathic retroperitoneal fibrosis patients: a retrospective study. Scand J Rheumatol. 2019;48:239-245.

26. Kermani TA, Crowson CS, Achenbach SJ, et al. Idiopathic retroperitoneal fibrosis: a retrospective review of clinical presentation, treatment, and outcomes. Mayo Clin Proc. 2011;86:297-303.

27. Moriconi D, Giannese D, Capecchi R, et al. Risk factors for relapse and longterm outcome of idiopathic retroperitoneal fibrosis. Clin Exp Nephrol. 2019;23:1147-1153.

28. Jadhav KK, Kumar V, Punatar CB, et al. Retroperitoneal fibrosis-clinical presentation and outcome analysis from urological perspective. Investig Clin Urol. 2017;58:371-377. doi:10.4111/ icu.2017.58.5.371

29. Labidi J, Ariba YB, Chargui S, et al. Retroperitoneal fibrosis: a retrospective review of clinical presentation, treatment and outcomes. Saudi J Kidney Dis Transpl. 2015;26:816-822. doi:10.4103/1319-2442.160226

30. Brandt AS, Kamper L, Kukuk S, Haage P, Roth S. Associated findings and complications of retroperitoneal fibrosis in 204 patients: results of a urological registry. $J$ Urol. 2011;185:526-531. doi:10.1016/j.juro.2010.09.105

31. Mizushima I, Kasashima S, Fujinaga Y, et al. Clinical and pathological characteristics of IgG4-related periaortitis/periarteritis and retroperitoneal fibrosis diagnosed based on experts' diagnosis. Ann Vasc Dis. 2019;12:460-472. doi:10.3400/avd.oa.19-00085

32. Castelein T, Coudyzer W, Blockmans D. IgG4-related periaortitis vs idiopathic periaortitis: is there a role for atherosclerotic plaque in the pathogenesis of IgG4-related periaortitis? Rheumatology (Oxford). 2015;54:1250-1256. doi:10.1093/rheumatology/keu462

33. Kasashima S, Kawashima A, Kasashima F, Endo M, Matsumoto Y, Kawakami K. Inflammatory features, including symptoms, increased serum interleukin-6, and C-reactive protein, in IgG4-related vascular diseases. Heart Vessels. 2018;33:1471-1481. doi:10.1007/s00380018-1203-8

34. Yamamoto M, Tabeya T, Naishiro Y, et al. Value of serum IgG4 in the diagnosis of IgG4-related disease and in differentiation from rheumatic diseases and other diseases. Mod Rheumatol. 2012;22:419-425. doi:10.3109/s10165-011-0532-6

35. Maritati F, Rocco R, Accorsi Buttini E, et al. Clinical and prognostic significance of serum IgG4 in chronic periaortitis. An analysis of 113 patients. Front Immunol. 2019;10:693. doi:10.3389/fimmu.2019.00693

36. Salvarani C, Pipitone N, Versari A, et al. Positron emission tomography (PET): evaluation of chronic periaortitis. Arthritis Rheum. 2005;53:298-303. doi:10.1002/art.21074

37. Fernando A, Pattison J, Horsfield C, et al. Fluorodeoxyglucose positron emission tomography in the diagnosis, treatment stratification, and monitoring of patients with retroperitoneal fibrosis: a prospective clinical study. Eur Urol. 2017;71:926-933.

38. Vaglio A, Greco P, Versari A, et al. Post-treatment residual tissue in idiopathic retroperitoneal fibrosis: active residual disease or silent "scar"? A study using 18F fluorodeoxyglucose positron emission tomography. Clin Exp Rheumatol. 2005;3:231-234.

39. Ebbo M, Grados A, Guedj E, et al. Usefulness of 2-[18F]-fluoro -2-deoxy-D-glucose-positron emission tomography/computed tomography for staging and evaluation of treatment response in IgG4-related disease: a retrospective multicenter study. Arthritis Care Res (Hoboken). 2014;66:86-96. doi:10.1002/acr.22058
40. Saeki T, Nishi S, Imai N, et al. Clinicopathological characteristics of patients with IgG4-related tubulointerstitial nephritis. Kidney Int. 2010;78:1016-1023. doi:10.1038/ki.2010.271

41. Raissian Y, Nasr SH, Larsen CP, et al. Diagnosis of IgG4-related tubulointerstitial nephritis. J Am Soc Nephrol. 2011;22:1343-1352. doi:10.1681/ASN.2011010062

42. Saeki T, Kawano M, Mizushima I, et al. The clinical course of patients with IgG4-related kidney disease. Kidney Int. 2013;84:826-833. doi:10.1038/ki.2013.191

43. Fry AC, Singh S, Gunda SS, et al. Successful use of steroids and ureteric stents in 24 patients with idiopathic retroperitoneal fibrosis: a retrospective study. Nephron Clin Pract. 2008;108:c213-c220. doi:10.1159/000119715

44. Ku JH, Lee SW, Jeon HG, et al. Percutaneous nephrostomy versus indwelling ureteral stents in the management of extrinsic ureteral obstruction in advanced malignancies: are there differences? Urology. 2004;64:895-899. doi:10.1016/j.urology.2004.06.029

45. Yamamoto M, Takahashi H, Shinomura Y. Mechanisms and assessment of IgG4-related disease: lessons for the rheumatologist. Nat Rev Rheumatol. 2014;10:148-159. doi:10.1038/nrrheum.2013.183

46. van Bommel EF, Siemes C, Hak LE, van der Veer SJ, Hendriksz TR. Long-term renal and patient outcome in idiopathic retroperitoneal fibrosis treated with prednisone. Am $J$ Kidney Dis. 2007;49:615-625. doi:10.1053/j.ajkd.2007.02.268

47. Mizushima I, Inoue D, Yamamoto $M$, et al. Clinical course after corticosteroid therapy in IgG4-related aortitis/periaortitis and periarteritis: a retrospective multicenter study. Arthritis Res Ther. 2014;16: R156. doi:10.1186/ar4671

48. Kamisawa T, Shimosegawa T, Okazaki K, et al. Standard steroid treatment for autoimmune pancreatitis. Gut. 2009;58:1504-1507. doi:10.1136/gut.2008.172908

49. Masamune A, Nishimori I, Kikuta K, et al. Randomised controlled trial of long-term maintenance corticosteroid therapy in patients with autoimmune pancreatitis. Gut. 2017;66:487-494.

50. Kubota K, Kamisawa T, Okazaki K, et al. Low-dose maintenance steroid treatment could reduce the relapse rate in patients with type 1 autoimmune pancreatitis: a long-term Japanese multicenter analysis of 510 patients. J Gastroenterol. 2017;52:955-964. doi:10.1007/s00535-016-1302-1

51. Vaglio A, Palmisano A. Treatment of Retroperitoneal Fibrosis; 2021. Available from: https://www.uptodate.com/contents/treatment-ofretroperitoneal-fibrosis?search $=$ treatment $\% 20$ of $\% 20$ retroperitoneal $\%$ 20fibrosis\&source $=$ search_result\&selectedTitle $=1 \sim 41 \&$ usage_type $=$ default\&display_rank=1. Accessed July 23, 2021.

52. Brandt AS, Kamper L, Kukuk S, et al. Tamoxifen monotherapy in the treatment of retroperitoneal fibrosis. Urol Int. 2014;93:320-325. doi:10.1159/000357814

53. Moroni G, Gallelli B, Banfi G, Sandri S, Messa P, Ponticelli C. Longterm outcome of idiopathic retroperitoneal fibrosis treated with surgical and/or medical approaches. Nephrol Dial Transplant. 2006;21:2485-2490. doi:10.1093/ndt/gfl228

54. van Bommel EF, Pelkmans LG, van Damme H, et al. Long-term safety and efficacy of a tamoxifen-based treatment strategy for idiopathic retroperitoneal fibrosis. Eur J InternMed. 2013;24:444-450. doi:10.1016/j.ejim.2012.11.010

55. Vaglio A, Palmisano A, Alberici F, et al. Prednisone versus tamoxifen in patients with idiopathic retroperitoneal fibrosis: an open-label randomized controlled trial. Lancet. 2011;378:338-346. doi:10.1016/S0140-6736(11)60934-3

56. Alberici F, Palmisano A, Urban ML, et al. Methotrexate plus prednisone in patients with relapsing idiopathic retroperitoneal fibrosis. Ann Rheum Dis. 2013;72:1584-1586. doi:10.1136/annrheumdis-2013-203267

57. Binder M, Uhl M, Wiech T, et al. Cyclophosphamide is a highly effective and safe induction therapy in chronic periaortitis: a long-term follow-up of 35 patients with chronic periaortitis. Ann Rheum Dis. 2012;71:311-312. doi:10.1136/annrheumdis-2011200148 
58. Průcha M, Kolombo I, Štádler P. Combination of steroids and azathioprine in the treatment of Ormond's disease - a single centre retrospective analysis. PragueMed Rep. 2016;117:34-41. doi:10.14712/23362936.2016.3

59. Scheel PJ Jr, Feeley N, Sozio SM. Combined prednisone and mycophenolate mofetil treatment for retroperitoneal fibrosis: a case series. Ann Intern Med. 2011;154:31-36. doi:10.7326/0003-4819-1541-201101040-00005

60. Maritati F, Corradi D, Versari A, et al. Rituximab therapy for chronic periaortitis. Ann Rheum Dis. 2012;71:1262-1264. doi:10.1136/ annrheumdis-2011-201166

61. Vaglio A, Catanoso MG, Spaggiari L, et al. Interleukin-6 as an inflammatory mediator and target of therapy in chronic periaortitis. Arthritis Rheum. 2013;65:2469-2475. doi:10.1002/art.38032

62. Della-Torre E, Campochiaro C, Bozzolo EP, et al. Methotrexate for maintenance of remission in IgG4-related disease. Rheumatology (Oxford). 2015;54:1934-1936. doi:10.1093/rheumatology/kev244

63. Yunyun F, Yu C, Panpan Z, et al. Efficacy of Cyclophosphamide treatment for immunoglobulin G4-related disease with addition of glucocorticoids. Sci Rep. 2017;7:6195. doi:10.1038/s41598-01706520-5

64. Yunyun F, Yu P, Panpan Z, et al. Efficacy and safety of low dose Mycophenolate mofetil treatment for immunoglobulin G4-related disease: a randomized clinical trial. Rheumatology (Oxford). 2019;58:52-60. doi:10.1093/rheumatology/key227

65. de Pretis N, Amodio A, Bernardoni L, et al. Azathioprine maintenance therapy to prevent relapses in autoimmune pancreatitis. Clin Transl Gastroenterol. 2017;8:e90. doi:10.1038/ctg.2017.17

66. Fukui S, Kawaai S, Nakai T, et al. Effectiveness and safety of mizoribine for the treatment of IgG4-related disease: a retrospective cohort study. Rheumatology (Oxford). 2021. keab235. doi:10.1093/ rheumatology/keab235.

67. Carruthers MN, Topazian MD, Khosroshahi A, et al. Rituximab for IgG4-related disease: a prospective, open-label trial. Ann Rheum Dis. 2015;74:1171-1177. doi:10.1136/annrheumdis-2014-206605

68. Wallwork R, Wallace Z, Perugino C, Sharma A, Stone JH. Rituximab for idiopathic and IgG4-related retroperitoneal fibrosis. Medicine (Baltimore). 2018;97:e12631. doi:10.1097/MD.0000000000012631

69. Urban ML, Maritati F, Palmisano A, et al. Rituximab for chronic periaortitis without evidence of IgG4-related disease: a long-term follow-up study of 20 patients. Ann Rheum Dis. 2020;79:433-434. doi:10.1136/annrheumdis-2019-216258

70. Mertens S, Zeegers AG, Wertheimer PA, Hendriksz TR, van Bommel EF. Efficacy and complications of urinary drainage procedures in idiopathic retroperitoneal fibrosis complicated by extrinsic ureteral obstruction. Int J Urol. 2014;21:283-288. doi:10.1111/ iju. 12234
71. Okumura A, Murakami K, Nozaki T, Fuse H. Laparoscopic ureterolysis for idiopathic retroperitoneal fibrosis. Int $J$ Urol. 2005;12:1079-1081. doi:10.1111/j.1442-2042.2005.01214.x

72. Stein RJ, Patel NS, Quinn K, et al. Laparoscopic ureterolysis with omental wrap for idiopathic retroperitoneal fibrosis. BJU Int. 2010;106:703-707. doi:10.1111/j.1464-410X.2009.09186.X

73. Hart PA, Kamisawa T, Brugge WR, et al. Long-term outcomes of autoimmune pancreatitis: a multicentre, international analysis. Gut. 2013;62(12):1771-1776. doi:10.1136/gutjnl-2012-303617

74. Kawa S, Hamano H, Ozaki Y, et al. Long-term follow-up of autoimmune pancreatitis: characteristics of chronic disease and recurrence. Clin Gastroenterol Hepatol. 2009;7:S18-22. doi:10.1016/j.cgh.2009.07.041

75. Wallace ZS, Mattoo H, Mahajan VS, et al. Predictors of disease relapse in IgG4-related disease following rituximab. Rheumatology (Oxford). 2016;55:1000-1008. doi:10.1093/rheumatology/kev438

76. Yamamoto M, Nojima M, Takahashi H, et al. Identification of relapse predictors in IgG4-related disease using multivariate analysis of clinical data at the first visit and initial treatment. Rheumatology (Oxford). 2015;54:45-49. doi:10.1093/rheumatology/keu228

77. Shirakashi M, Yoshifuji H, Kodama Y, et al. Factors in glucocorticoid regimens associated with treatment response and relapses of IgG4-related disease: a multicentre study. Sci Rep. 2018;8(1):10262. doi:10.1038/s41598-018-28405-X

78. Mizushima I, Tsuge S, Fujisawa Y, et al. Different factors underlie recurrent and de novo organ involvement in immunoglobulin G4-related disease. Rheumatology (Oxford). 2020;59:513-518.

79. Tajima M, Hiroi Y, Takazawa Y, et al. Immunoglobulin G4-related multiple systemic aneurysms and splenic aneurysm rupture during steroid therapy. Hum Pathol. 2014;45:175-179. doi:10.1016/j. humpath.2013.07.035

80. Kasashima S, Kawashima A, Kasashima F, et al. Immunoglobulin G4-related periaortitis complicated by aortic rupture and aortoduodenal fistula after endovascular AAA repair. $J$ Endovasc Ther. 2014;21:589-597. doi:10.1583/14-4670R.1

81. Ozawa M, Fujinaga Y, Asano J, et al. Clinical features of IgG4-related periaortitis/periarteritis based on the analysis of 179 patients with IgG4-related disease: a case-control study. Arthritis Res Ther. 2017;19:223. doi:10.1186/s13075-017-1432-8
The International Journal of Nephrology and Renovascular Disease is an international, peer-reviewed open-access journal focusing on the pathophysiology of the kidney and vascular supply. Epidemiology, screening, diagnosis, and treatment interventions are covered as well as basic science, biochemical and immunological studies. The manuscript management system is completely online and includes a very quick and fair peer-review system, which is all easy to use. Visit http://www.dovepress.com/testimonials.php to read real quotes from published authors. 